\title{
History-based spectrum sensing in CR-VANETs
}

\author{
Shahid H Abbassi ${ }^{1 *}$, ljaz M Qureshi ${ }^{1}$, Hameer Abbasi ${ }^{2}$ and Bahman R Alyaie ${ }^{1}$
}

\begin{abstract}
Cognitive radio spectrum sensing is an important issue in today's emerging communication techniques. Similarly, vehicular networks are vital considering the increasing traffic on roads and fatal accidents. Efficient networks can reduce the road accidents and enhance the suitability of communication between vehicles and with fixed infrastructure. Different techniques like independent spectrum sensing and various forms of cooperative techniques have been proposed in the near past. We propose a sensing technique which prepares a database for small road segments, time slots for the hours of the day, and different frequencies of the spectrum based on the sensing of vehicles throughout the day. Based on this database, the future utilization of the spectrum is proposed. Simulations and results clearly indicate the success and usefulness of our proposed technique.
\end{abstract}

Keywords: DSRC; CCH; TV bands; RSU; IEEE 802.11p; WAVE

\section{Introduction}

Approximately 1.24 million people die every year and nearly 20 to 50 million are affected by fatal injuries due to road accidents around the globe according to a report published by the World Health Organization. The report further states that road accidents are the eighth leading cause of fatal injuries and may become the fifth leading cause if proper measures are not taken to reduce road accidents. Road traffic injuries are estimated to cost over US\$100 billion to low- and middle-income countries, which is an estimated 1-2\% of their gross annual product [1].

Vehicular ad hoc networks (VANETs), a special form of mobile ad hoc networks (MANETs), are a kind of pervasive networks (PN) which provide a number of services from a single access point to the road travelers. VANETs can greatly reduce road accidents and provide safety and entertainment services [2]. Interested readers may find the technical details of VANET frequency allocation in [3]. Dedicated short-range communication (DSRC) provides ways to propagate warnings to road travelers; details on this may be found in [4]. Long-Term Evolution (LTE) has also

\footnotetext{
* Correspondence: shahid.abbassi@mail.au.edu.pk

${ }^{1}$ Electrical Engineering Department, Air University, E-9, Islamabad, Pakistan Full list of author information is available at the end of the article
}

been used in place of DSRC in some places [5]. Details on the DSRC protocol stack can be found in [6].

According to an estimate by the US Department of Transportation (DoT), with the deployment of DSRC standard, about $82 \%$ of the road accidents can be reduced. It has been observed that road traffic is increasing day by day. For example, in the UK, the traffic has increased from 26 billion vehicle miles to nearly 70 billion vehicle miles from the year 1949 to 2012 [7]. This trend of increasing road traffic will cause increased need of communication among vehicles and between vehicles and road side units which will overburden the proposed DSRC spectrum. Therefore, other means for vehicular communication have to be explored to maintain a reliable, high-bandwidth, and low-latency transfer of messages within the vehicular infrastructure. Cognitive radios (CR) are an emerging technology, which may be exploited by vehicles to at least convey emergency messages on underutilized wireless frequencies such as TV bands (VHF and UHF), Wi-Fi, Wi-MAX, cellular phone, Bluetooth, and satellite communication [8]. Due to a regulatory framework, only licensed users are allowed to utilize the respective spectrum and no one else is allowed to use the spectrum resources on their own. In CR, unlicensed (secondary) users are allowed to borrow unutilized bandwidth from licensed (primary) users. 


\begin{tabular}{|l|l|l|l|l|l|l|l|l|l|l|l|l|l|l|l|l|l|l|}
\hline$N_{j}$ & $N_{F_{-}}$ & $N_{j}$ & & $N_{B}$ & $N_{j}$ & & $N_{j}$ & $N_{j}$ & $N_{M}$ & $N_{j}$ & & $N_{j}$ & & $N_{F}$ & $N_{j}$ & & $N_{B_{+}}$ & $N_{j}$ \\
$\infty$ & $\infty$ & $\infty$ & & $\infty$ & $\infty$ & & $\infty$ & $\infty$ & $\infty$ & $\infty$ & & $\infty$ & & $\infty$ & $\infty$ & & $\infty$ & $\infty$ \\
\hline
\end{tabular}

Fig. 1 Segment distribution in a cluster

Sharing of network resources continues in this fashion until primary users need more of the spectrum, in which case secondary users have to vacate the spectrum $[9,10]$. Cognitive radios can be built so they are smart enough to use parameters (such as carrier frequency, bandwidth, and transmission power) of that particular spectrum band and particular wireless technology [11].

It can be observed that some spectrum bands like TV channels have spatio-temporal patterns. During some hours of the day and in some areas, some channels are on while others are off. These patterns can be exploited to improve the efficiency of spectrum sensing by making it more directed. This paper utilizes the history of spatio-temporal and frequency patterns and proposes a spectrum sensing technique controlled by roadside units (RSUs) and coordinating nodes, which directs the vehicles to sense those channels first whose probability of availability is high during that particular slot.

The rest of the paper is organized as follows: Related work is in "Section 2." "Section 3" describes the proposed system model. "Section 4" explains the simulations conducted and results. "Section 5" concludes the paper.

\section{Related work}

As the safety of many road travelers and their passengers is at stake, algorithms for VANETs need to be designed with extreme care in order to avoid any unwanted incidents. Fawaz and Ghandour [12], Felice and Chowdhury [13], Kim and Oh [14], Husheng and Irick [15], Nyanhete et al. [16], and Rawat [17] propose stand-alone CR spectrum sensing techniques. Fawaz and Ghandour [12] propose a structure comprising RSUs, local acquisition and processing units (LAPUs), and vehicles. Decisions for spectrum holes are taken by RSUs and LAPUs, and CR technology is used to decrease the load on the control channel $(\mathrm{CCH})$ of the IEEE $802.11 \mathrm{p} /$ WAVE spectrum. Felice and Chowdhury [13] propose a scheme that each vehicle may be assigned a TV channel to sense and use it independently, and vehicles share the spectrum availability information among each other for future usage. This scheme has no coordination and cooperation and hence may cause interference with the primary user network in the case of any misdetection. In [14], it is proposed that vehicles may utilize Wi-Fi and ISM (2.4 or $5 \mathrm{GHz}$ ) bands in urban areas and may share the sensing information for future usage. In [15], a spectrum sensing scheme based on belief propagation

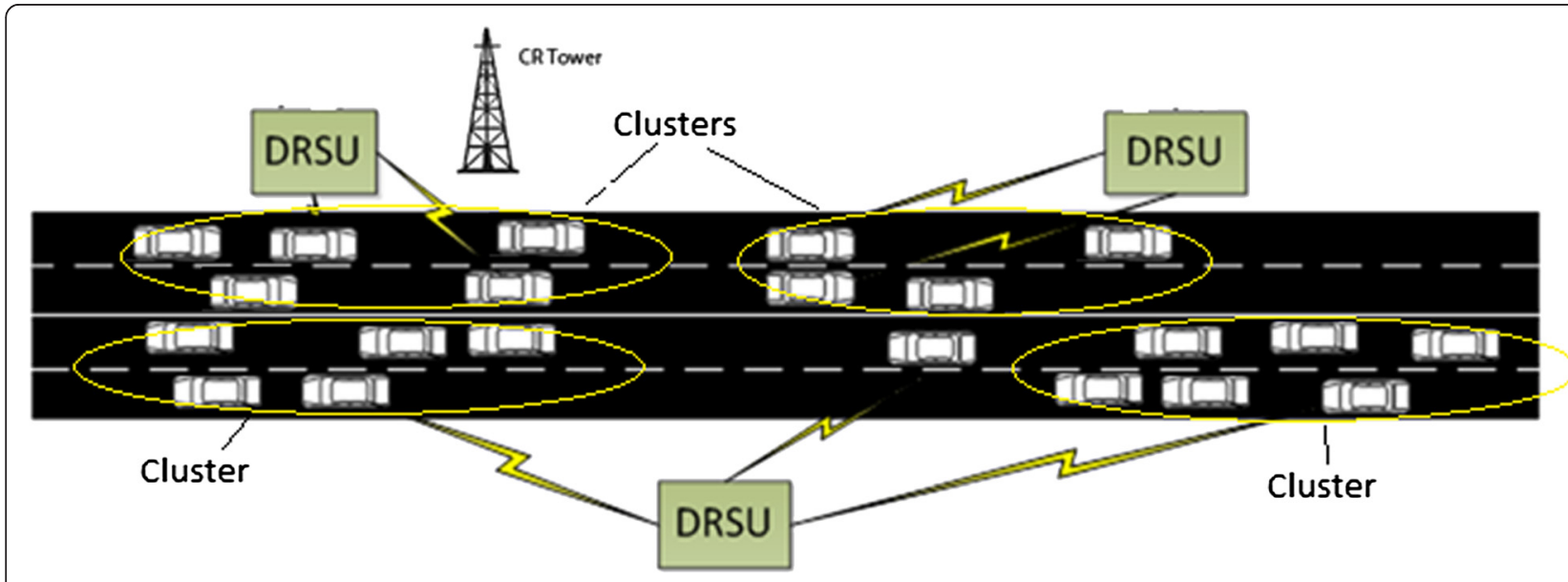

Fig. 2 Clusters in a RSU-based network 


\begin{tabular}{|l|l|l|l|l|lllll|l|l|l|}
\hline 1 & 0 & 1 & 1 & 0 & $\ldots$ & $\ldots$ & $\ldots$ & $\ldots$ & 0 & 1 & 0 \\
\hline \begin{tabular}{|l}
\hline \\
Fig. 3 Contents of a $T_{k}$ time slot
\end{tabular} & & & & & & & & & & & & \\
\hline
\end{tabular}

is proposed. In this scheme, every vehicle sends the information related to presence or absence of primary user signals in its range to its neighbors. Each vehicle based on received information and its own prediction detects and uses the spectrum. Also, in [16], every secondary user senses and uses the available spectrum holes on its own and also shares the information with neighbors. In [17], a three-state model has been proposed. The first state occurs if a hole is detected, the second if a primary user is present, and the third if a secondary user occupies the channel. This scheme also insists on individual sensing and utilization of the spectrum.

In [18], the authors have proposed a cooperative sensing Cog-V2V proposal in which every vehicle collects the sensing data and shares with the vehicles in its range on common $\mathrm{CCH}$. Roads are divided into segments and the spectrum horizon of each vehicle is defined. Every vehicle based on the received information and its own observations for the related road segment and frequency aggregates the data and finds the similarity function. It decides the availability of any channel based on the aggregated data and the similarity function. In [19], the authors have further enhanced the Cog-V2V framework by considering the impact of correlated shadowing on the sensing output. Based on correlation, the weight factor has been calculated and multiplied with the sensing binary output to get the decision of a primary user's presence or absence. These two papers do not take into account the temporal variations in the availability of any spectrum band in deciding the spectrum band as a hole. In [20], a new method has been proposed to improve the sensing performance over a time-variant multipath flat-fading (TVMFF) channel. A two-state Markov chain is used to check the changes of the PU state.

In [21], a novel sensing scheme combining the best of stand-alone and cooperative spectrum sensing has been proposed. A coordinating node senses the spectrum and forms a table of spectrum holes. It sends a list of empty slots (holes) to the requesting vehicle. The requesting vehicle then rescans the received holes and reconfirms the availability of the spectrum. If it finds a hole, it utilizes it for communication. In [22], a cluster-based scheme is proposed with each cluster having a cluster head. The cluster head senses the spectrum and assigns the spectrum holes to its cluster members. These papers do not include the shadowing, correlation, and distance factors as a hole for one vehicle may not be a hole for another due to a high dynamic environment. In [23], the authors have a division of the wideband spectrum into small sub-bands and assigning a sub-band to a group of vehicles to sense and utilize the provided spectrum. This paper does not take into consideration any criteria for assigning any sub-band to a particular group of vehicles. The assigned sub-band may or may not suit any

\begin{tabular}{|c|c|c|c|c|c|c|c|c|c|c|c|c|c|}
\hline Channels & \multicolumn{4}{|c|}{ Segment 1} & \multicolumn{4}{|c|}{ Segment 2} & $2 \ldots \ldots \ldots$ & \multicolumn{4}{|c|}{ Segment N } \\
\hline 1 & 0.4 & 0.32 & $\ldots .$. & 0.23 & 0.92 & 0.95 & $\ldots$. & 0.71 & …................... & 0.57 & 0.22 & $\ldots \ldots$ & 0.13 \\
\hline 2 & 0.56 & 0.29 & ..... & 0.68 & 0.64 & 0.31 & ..... & 0.69 & …….... & 0.74 & 0.82 & $\ldots \ldots$ & 0.66 \\
\hline .. & .. & .. & ..... & .. & .. & .. & ..... & .. & . & .. & .. & $\ldots \ldots$ & .. \\
\hline .. & .. & .. & ..... & .. & .. & .. & ..... & .. & $\ldots \ldots \ldots \ldots \ldots \ldots \ldots \ldots$ & .. & .. & $\ldots \ldots$ & .. \\
\hline $\mathrm{M}$ & 0.8 & 0.81 & ...... & 0.57 & 0.63 & 0.37 & $\ldots \ldots$ & 0.34 & $\ldots \ldots \ldots \ldots \ldots \ldots \ldots \ldots$ & 0.44 & 0.69 & $\ldots \ldots$ & 0.78 \\
\hline Slots & 1 & 2 & $\ldots \ldots$ & $\mathrm{K}$ & 1 & 2 & $\ldots \ldots$ & $\mathrm{K}$ & & 1 & 2 & $\ldots \ldots$ & $\bar{K}$ \\
\hline
\end{tabular}

Fig. 4 Database maintained by the RSUs 
Table $1 N_{\mathrm{M}}$ reply to $N_{\mathrm{j}}$

\begin{tabular}{llllll}
\hline Segment & Channels & & & & \\
\hline$S_{i}$ & 12 & 5 & 3 & 14 & 8 \\
$S_{i+1}$ & 7 & 5 & 1 & 2 & 9 \\
$S_{i+2}$ & 13 & 11 & 3 & 7 & 4 \\
$S_{i+3}$ & 3 & 12 & 15 & 8 & 6 \\
\hline
\end{tabular}

particular group of vehicles. In [24], multi-RAT technology has been discussed. It suggests usage of different technologies for different classes of service such as Wi-Fi for data exchange and Wi-MAX for video conferencing. In this paper, no spectrum sensing technique or any cooperative scheme has been discussed. In [25], a two-user model has been proposed. In this model, one user acts as a relay sending the information regarding the primary user's presence or absence to the other user. In this model, two cooperative sensing schemes have been proposed. In the first scheme, one user detects the primary user's presence or absence and relays to the other user through a central controller. In the second scheme (in order to reduce the detection time), the amplify and forward (AF) protocol has been used.

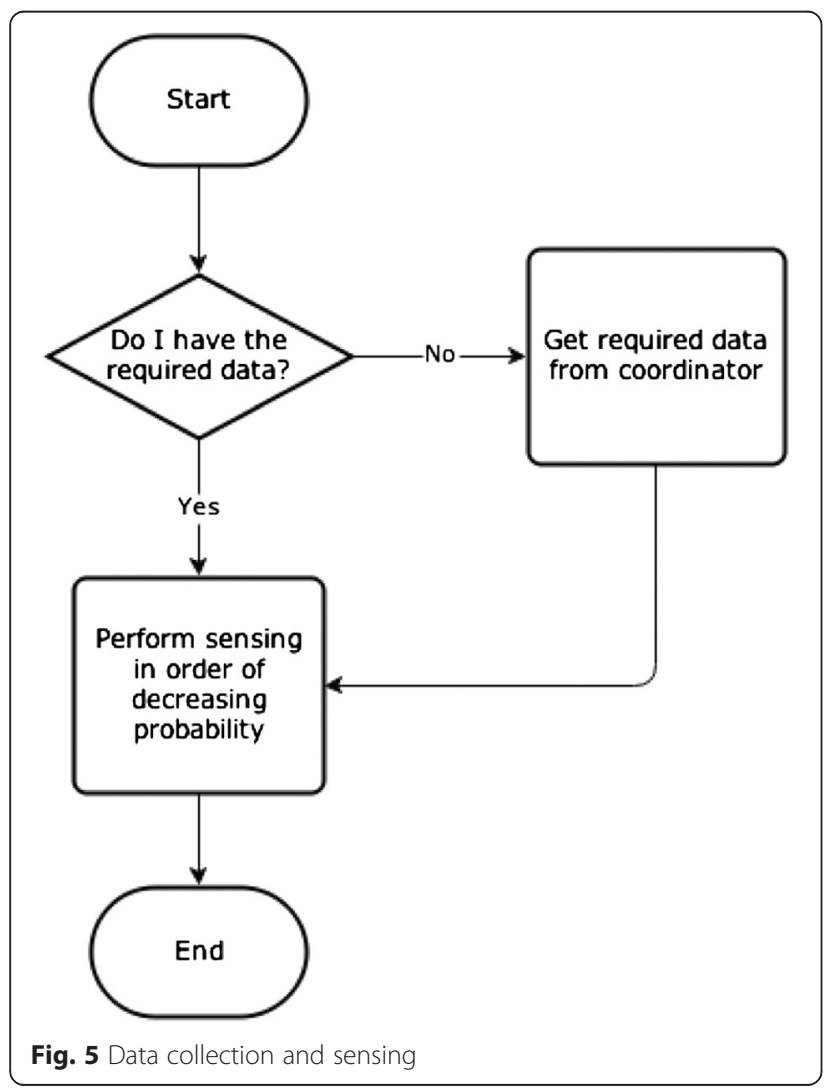

In this paper, we propose a technique that uses more than one coordinator. Every coordinator collects the localized availability information and sends it through the main coordinator to RSUs. RSUs update the data for each road segment, frequency, and time segment. This data is reused during the sensing of the spectrumgiving channels that have a higher probability of availability priority in sensing. VANET has a high dynamic node environment so the proposed techniques discussed in related work do not exactly suit it.

\section{Proposed system model}

Our proposed system relates to suburban areas or highways. It is understood that RSUs are installed at proper distances on the sides of highways. For efficient and systematic communication among vehicles and between vehicles and infrastructure, directional RSUs (DRSUs) shall be installed as proposed in [26] and [27]. As DSRUs have separate processors and storage locations for each direction, cluster management can be achieved easily because clusters remain intact for longer times on separate directions of any highway. Vehicles are managed in clusters on each direction and each cluster has three coordinators: $N_{\mathrm{M}}$ (main coordinator), $N_{\mathrm{F}}$ (front coordinator), and $N_{\mathrm{B}}$ (back

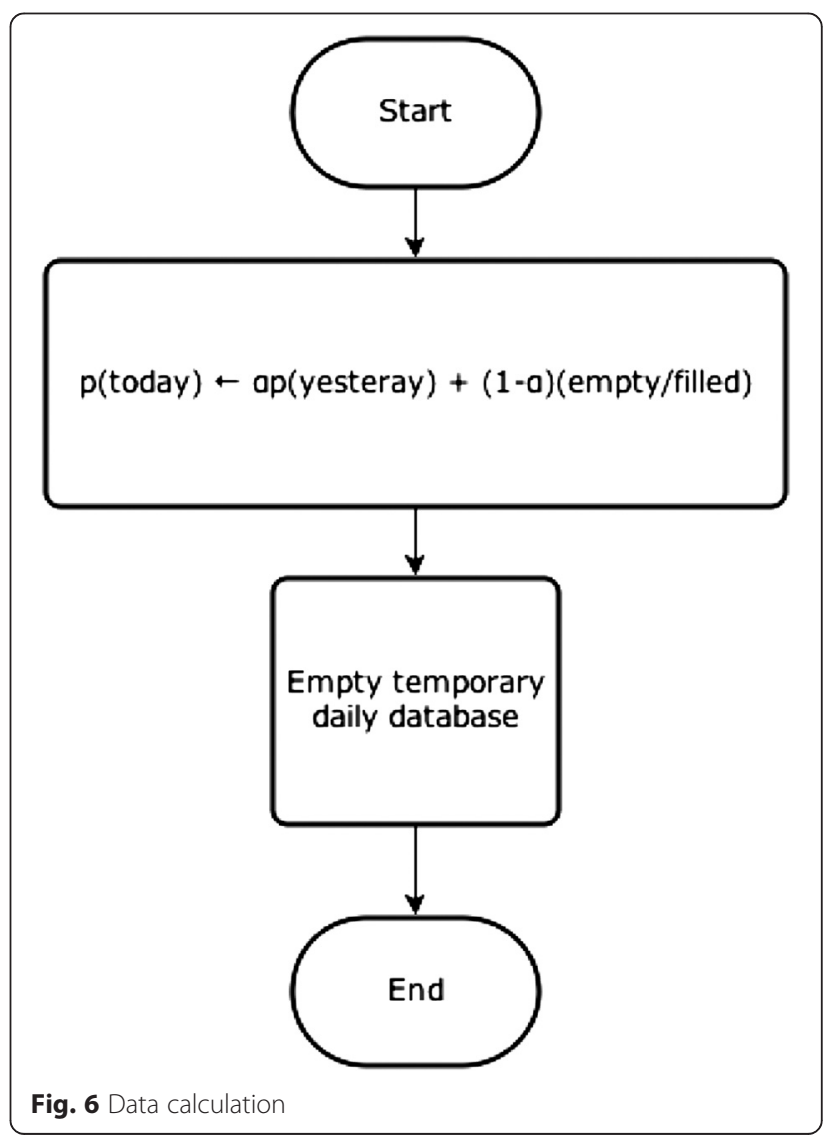




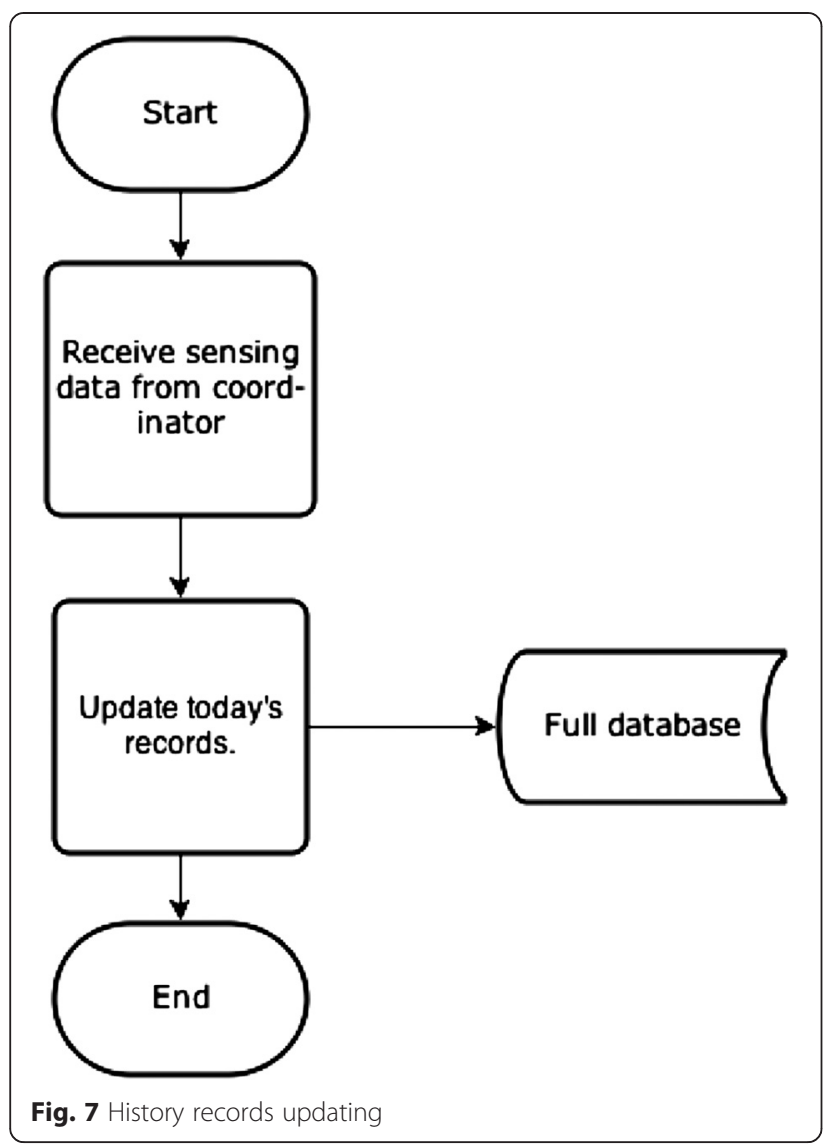

coordinator) for $\mathrm{CR}$ spectrum sensing and coordination activities as proposed in [28]. The cognitive radio spectrum, especially the TV spectrum, is divided into $\mathrm{M}$ channels. Communication range of $N_{\mathrm{M}}$ is divided into 15 equal distance segments denoted by $S_{i}$ $[18,19]$ as shown in Fig. 1. $N_{\mathrm{M}}$ is moving in distance segment $S_{\mathrm{C}}, N_{\mathrm{F}}$ in $S_{\mathrm{C}+5}$, and $N_{\mathrm{B}}$ in $S_{\mathrm{C}-5}$, which are progressively changing as vehicles move forward and jump into the next distance segment. $S_{\mathrm{bc}}$ is the third last segment of the backward cluster and carries the front coordinator $N_{\mathrm{F}-}$ of that cluster. $S_{\mathrm{fc}}$ is the third segment of the front cluster and carries the back coordinator $N_{\mathrm{B}+}$ of that cluster. The segments $S_{\mathrm{C}-6}$ and $S_{\mathrm{C}-7}$ are overlapping segments between the backward and current cluster, whereas the segments $S_{\mathrm{C}}+6$ and $S_{\mathrm{C}+7}$ are overlapping segments between the front and current clusters. It is important to mention here that the highway considered here is busy, but as traffic conditions and patterns change dynamically and maybe some of the segments contain no vehicles (in shallow traffic conditions), then positions of back and front coordinators may shift one or two segments front or backwards. The selection of the coordinators is well defined in [28].

Every vehicle $N_{\mathrm{j}}$ sends and receives the control information on the CCH of 5.9-GHz IEEE 802.11p/DSRC spectrum. Vehicles in segments $S_{C}-2$ to $S_{C}+2$ send and receive control messages to $N_{\mathrm{M}}$; vehicles in segments $S_{\mathrm{C}+3}$ to $S_{\mathrm{C}+7}$ to $N_{\mathrm{F}}$; and vehicles in segments $S_{\mathrm{C}-7}$ to $S_{\mathrm{C}-3}$ to $N_{\mathrm{B}}$. Vehicles in segments $S_{\mathrm{C}+6}$ and $S_{\mathrm{C}+7}$ also send and receive control messages to $N_{\mathrm{B}+}$, and vehicles in segments $S_{\mathrm{C}-7}$ and $S_{\mathrm{C}-6}$ also send and receive control messages to $N_{\mathrm{F}-}$ to receive the information on next segments from the front cluster or to provide information to the backward cluster. Further, every vehicle $N_{\mathrm{j}}$ is equipped with a GPS (Global Positioning System) chip in order to locate its position and track the roads and intended destination.

\subsection{History updating}

Every vehicle $N_{\mathrm{j}}$ (secondary user) senses the M channels of a TV spectrum every $T_{\mathrm{s}}$ second using the energy detection technique in order to identify the presence or absence of licensed users (primary users) in the

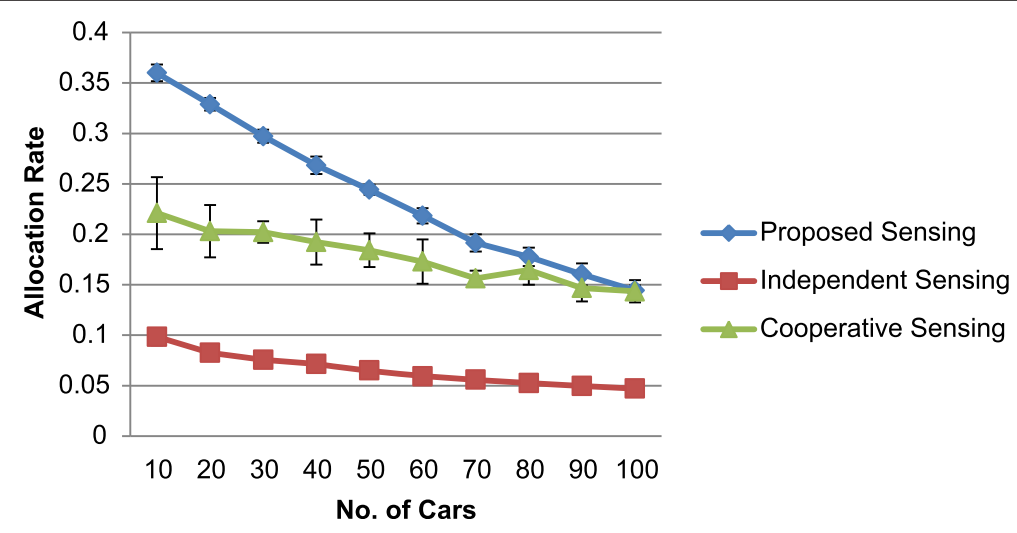

Fig. 8 Allocation rate vs no. of cars 


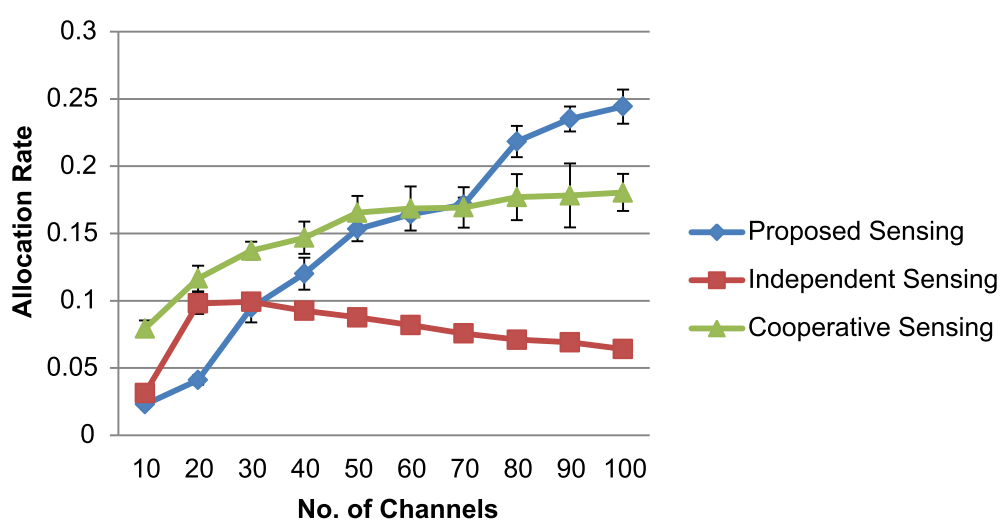

Fig. 9 Allocation rate vs no. of channels

spectrum. Binary hypothesis test is conducted, indicating $H_{1}$ for the presence of a primary user and $H_{0}$ for the absence of a primary user as given by Eq. 1 [28].

$$
r_{i}(t)=\left\{\begin{array}{lr}
\operatorname{Re}\left\{\left[h_{\mathrm{c}} S_{\mathrm{LP}}(t)+n_{\mathrm{LP}}(t)\right] e^{j 2 \pi f_{*}^{i} t}\right\}, & H_{1} \\
\operatorname{Re}\left\{n_{\mathrm{LP}}(t) e^{j 2 \pi f_{*}^{i} t}\right\}, & H_{0}
\end{array}\right.
$$

where $\operatorname{Re}\{$.$\} is the real part of the received complex$ waveform and $f_{*}^{i}$ s the carrier frequency of the primary channel with $i=1,2,3, \ldots$ M representing the channel sensed and "indicates V or U for VHF or UHF respectively. If the secondary user is occupying the primary network, then we use the secondary user pilot carrier frequency for the detection $f_{s}^{i} . S_{\mathrm{LP}}(t)$ is the equivalent low-pass representation of the detected primary or secondary user signal. $n_{\mathrm{LP}}(t)$ is the additive white Gaussian noise with 0 mean.

Every $N_{\mathrm{j}}$ after sensing sends a sense message containing the results (binary 1 for $H_{1}$ and binary 0 for
$H_{0}$ ) for every channel it sensed along with its coordinates indicating the distance segment and also its identity in order to avoid duplication of messages on $\mathrm{CCH}$ to $N_{\mathrm{M}}, N_{\mathrm{F}}$, or $N_{\mathrm{B}}$ depending on its location in the cluster. Each entry in the list contained in the message is formatted as follows.

$$
<V_{\text {id }} X Y \text { CH RES Time }>
$$

where $V_{\text {id }}$ is the vehicle identity, $X$ and $Y$ are the coordinates received from GPS to locate the segment, $\mathrm{CH}$ is the channel sensed from 1 to $M, R E S$ is the sensing result ( 1 or 0$)$, and Time is the sensing time. It shall be noted that $N_{\mathrm{j}}$ in $S_{\mathrm{C}+6}$ to $S_{\mathrm{C}+7}$ and $S_{\mathrm{C}-7}$ to $S_{\mathrm{C}-6}$ also sends the sense message to $N_{\mathrm{B}+}$ and $N_{\mathrm{F}-}$ respectively in order to be aware of the future locations on the path. Figure 2 shows the cluster formation in a RSU-based network. Instead of RSUs, we have provided DRSUs as proposed in [26].

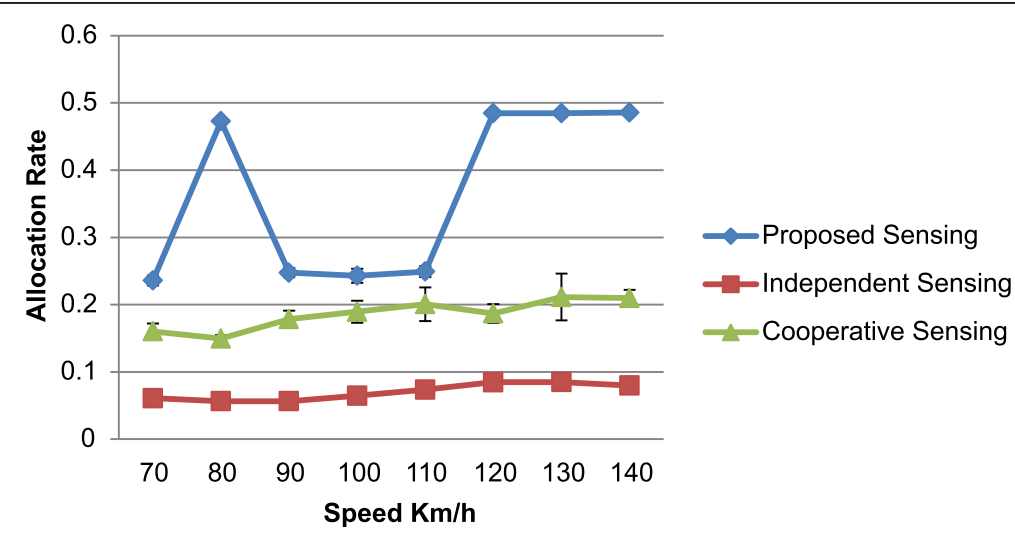

Fig. 10 Allocation rate vs speed 


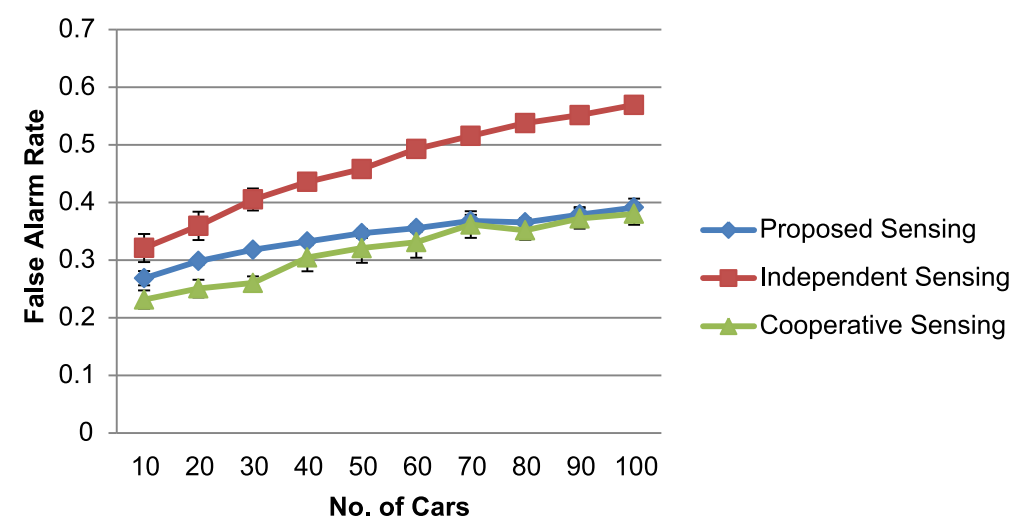

Fig. 11 False alarm rate vs no. of cars

$N_{\mathrm{M}}, N_{\mathrm{F}}$, or $N_{\mathrm{B}}$ on receipt of sensed messages from the vehicles for one $T_{\mathrm{s}}$ interval compiles the results based on majority decision if more than one result is obtained from one segment $S_{i}$ for the same channel $f$. Results for the space, time, and frequency are finalized and sent to the main coordinator for onward submission to the RSU. A sample entry in the list contained in message sent to RSU is of the format as given below. Output indicates the binary 1 for primary or secondary user presence and binary 0 for absence. RSUs on receipt of update message update in a spatial-time-frequency database.

$$
<S_{i} T_{\mathrm{s}} \text { f Output }>
$$

\subsection{History preservation}

RSU divides the $24 \mathrm{~h}$ of the day into $K T_{k}$ time slots. Each $T_{k}$ time slot is further subdivided into $S T_{\mathrm{s}}$ time intervals. For each $T_{\mathrm{s}}$, data entries are collected from the clusters for every distance segment and every channel. One $T_{k}$ time slot containing $S T_{\mathrm{s}}$ time intervals is represented in Fig. 3.

Probability for the availability of the channel is computed by the RSU based on the number of zeros in the $T_{k}$ time slot, and a database for $K$ time slots, each channel, and each segment is stored. Database entries computed and stored by the RSUs will look like as shown in Fig. 4.

$$
p_{\text {av }}=\frac{\text { Total number of zeros in } T_{k}}{S}
$$

RSUs exchange the database with each other, and each RSU stores the database for the segments covered by its basic service set (BSS) and also for the segments covered by the BSS of the next RSU. This will enable the vehicles to utilize the spectrum proactively on their future path to the destination.

\subsection{History utilization}

Each RSU sorts the database in order of high probability for the next time slots and shares the information

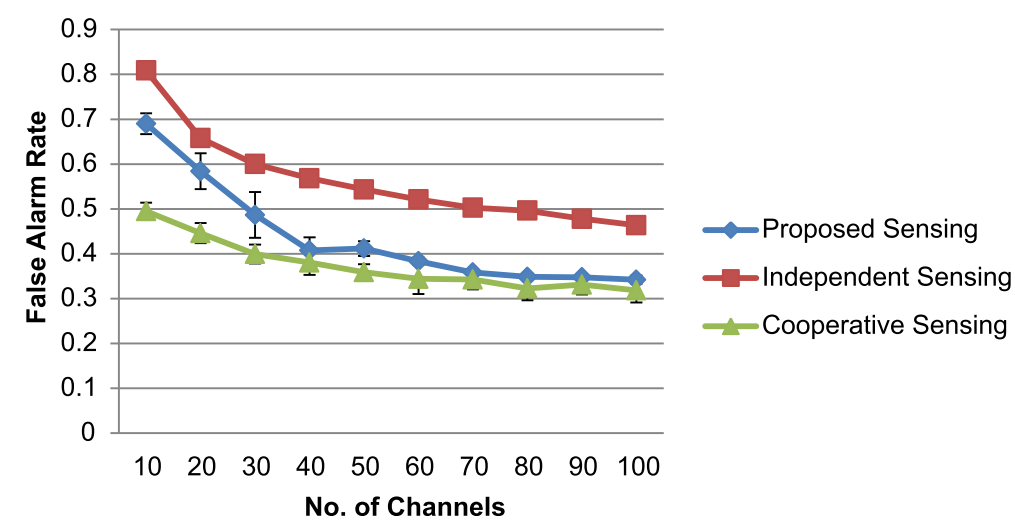

Fig. 12 False alarm rate vs no. of channels 


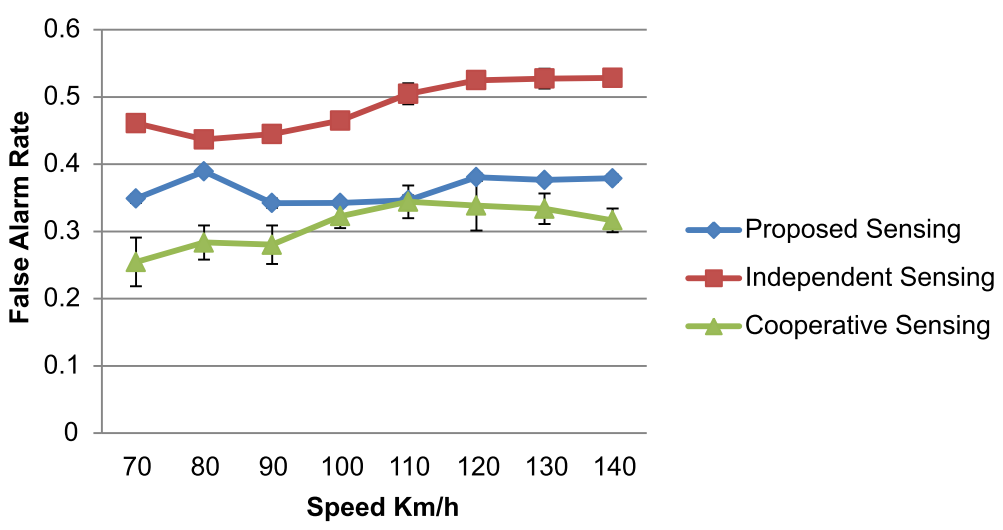

Fig. 13 False alarm rate vs speed

with main coordinators of the clusters for the segments covered by that main coordinator and its future path. The main coordinator $N_{\mathrm{M}}$ shares the information with $N_{\mathrm{F}}$ and $N_{\mathrm{B}}$. All three coordinators sense the channels as per procedure discussed in [3], compile the results, and send back to the main coordinator. $N_{\mathrm{M}}$, on receipt of results from itself and the other two coordinators, compiles the results based on current availability and high availability probability received from RSU for the segments in its coverage range. On receipt of $C R$ spectrum utilization request from any $N_{\mathrm{j}}$ moving in segment $S_{i}$ and time slot $T_{k}, N_{\mathrm{M}}$ sends back the channel numbers in order of higher probability of availability to the lower one for the next distance segments and time slot $T_{k}$. A sample format of reply sent by $N_{\mathrm{M}}$ to the requesting $N_{\mathrm{j}}$ will look like as shown in Table 1.

$N_{\mathrm{j}}$, on receipt of reply from the $N_{\mathrm{M}}$ as per format in Table 1, rescans the channels in order of higher to lower probability and utilizes the first available channel for its communication and continuously scans the channels for the next segments and switches to other channels if required on its way to the destination.

\section{Simulation and results}

Simulations have been performed using Visual C\#. The spectrum sensing mechanism used is as presented in [28], with the alterations that the Hata model for suburban areas was used as the long-scale fading model, and Rayleigh fading was used as the smallscale fading model. The mechanism for the movement of vehicles as well as other parameters has been lifted from these papers as well. The only thing added on is spatio-temporal history, databases, and predictive sensing as discussed below. As shown in Fig. 5, whenever a vehicle wants to use the CR spectrum, it checks the sensing data it has available in its own database, corresponding to the current time and space segment. If unavailable, it requests new history data for its time segment and future segments and, similarly for space, from the coordinators.

Similarly, if the coordinator finds that its own data is inadequate, it requests data from the RSU, for its entire communication range and then some more, so it will not have to request new data too much.

Sensing is performed as detailed above, but with a minor difference: Channels with a higher probability

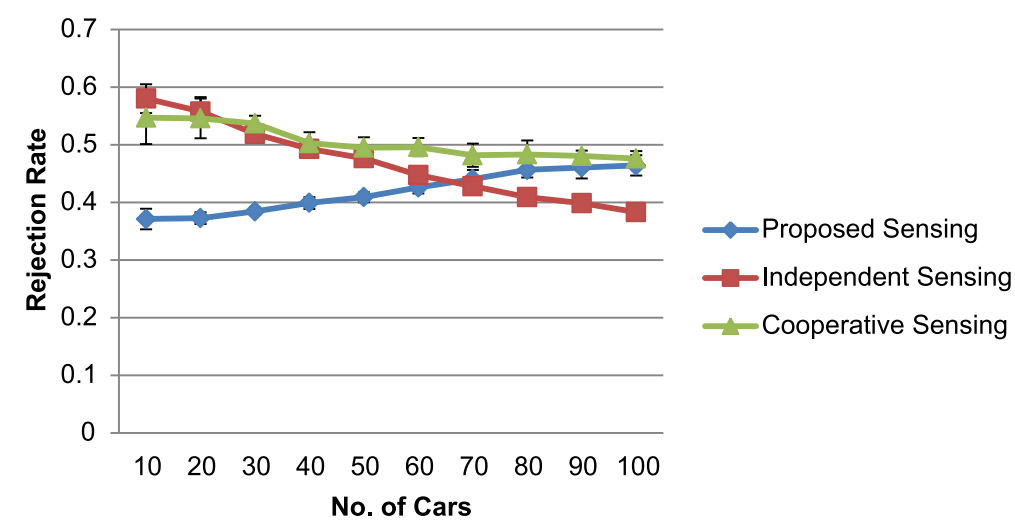

Fig. 14 Rejection rate vs no. of cars 


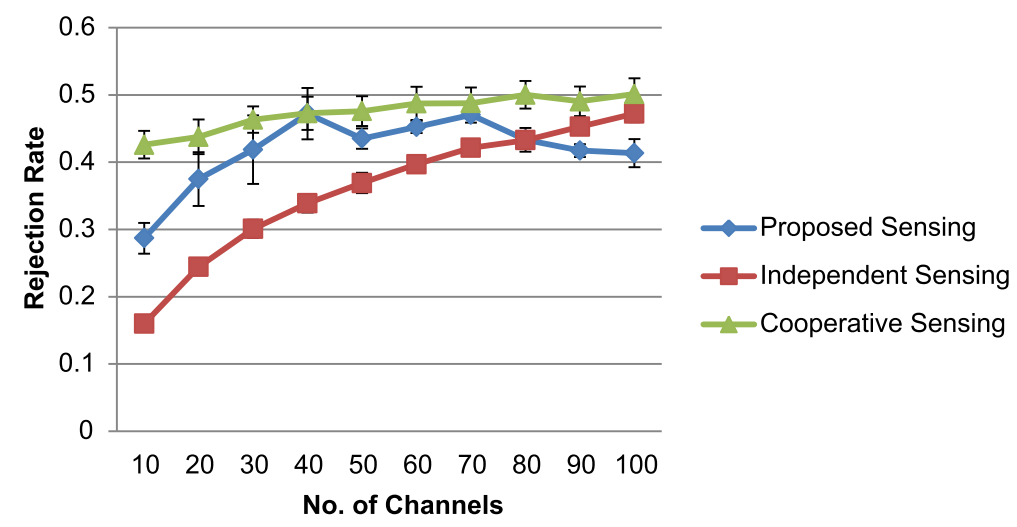

Fig. 15 Rejection rate vs no. of channels

of being empty (also for a longer amount of time) in the given spatio-temporal slot are sensed first; with the first available channel being occupied.

This process is then reversed, with simple (non-coordinating) vehicles sending data to the coordinators and the coordinators sending it to the RSUs, with the RSUs updating daily records. Figures 6 and 7 detail the process.

There were a number of different parameters used during the simulation, which are listed as follows: the vehicle communication range was $240 \mathrm{~m}$, and the average car speed varied from 80 to $120 \mathrm{~km} / \mathrm{h}$, with each individual car varying its speed between 5 to 10 $\%$. Noise power was $4.83228 \times 10^{-14} \mathrm{~W}$ (measured by the popular formula for additive white Gaussian noise $B \mathrm{k} T$, where $B$ is the bandwidth (7 $\mathrm{MHz}$ for a typical TV channel), $k$ is the Boltzmann constant, and $T$ is the noise temperature (we used $500 \mathrm{~K}$ )). The threshold used to measure if another user was present was $7 \times$ $10^{-11} \mathrm{~W}$. The Hata model for suburban areas was used for fading, with the height of the transmitting antenna $=50 \mathrm{~m}$, the height of the receiving antenna $=$ $1.5 \mathrm{~m}$, and the center frequency $=1.5 \mathrm{GHz}$. Cars would communicate for 20 to $30 \mathrm{~ms}$ and stay "silent" for 0.4 to $0.5 \mathrm{~s}$. The patch of road was $2 \mathrm{~km}$ long.

A number of different metrics have been observed in these simulations. The most important of these metrics are allocation rate, false alarms, misdetections, rejection rate, and forced leaves. Allocation rate is the ratio of the number of successful allocations to the number of total attempts; hence, it provides a clear picture of the success of the algorithm. False alarm indicates an empty channel detected busy, and the ratio is the number of false alarms to the number of total attempts. Lower false alarms are better for efficient resource utilization but are not harmful for the spectrum and primary user. Misdetection indicates a busy channel detected empty. Misdetections are very harmful as it leads to interference with the primary user signals and are needed to be eliminated. Our algorithm as discussed in [28] and has successfully eliminated misdetections. Rejection rate tells the number of unsuccessful attempts to the total number of attempts. A lower rejection rate is successfulness of the algorithm. Forced leave ratio indicates how many

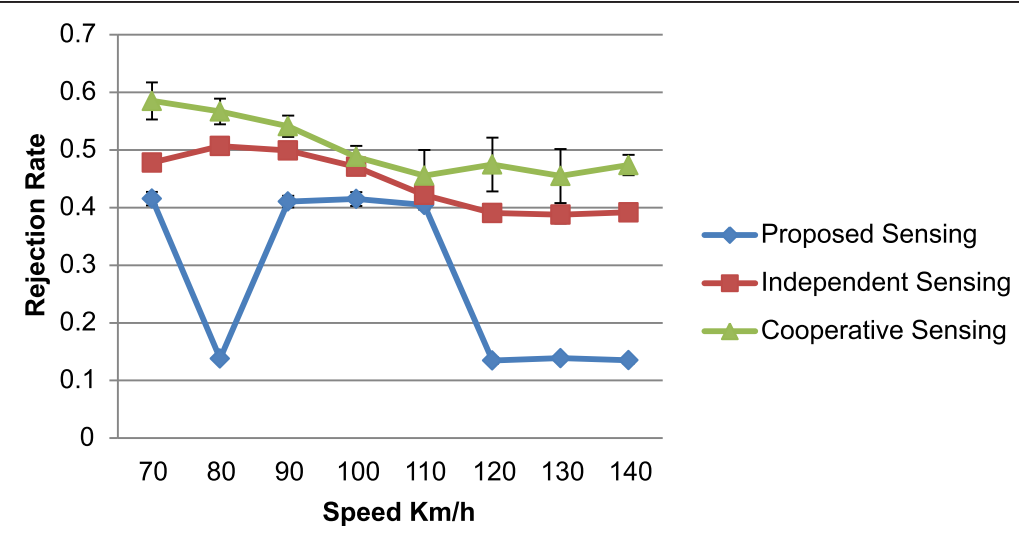

Fig. 16 Rejection rate vs speed 


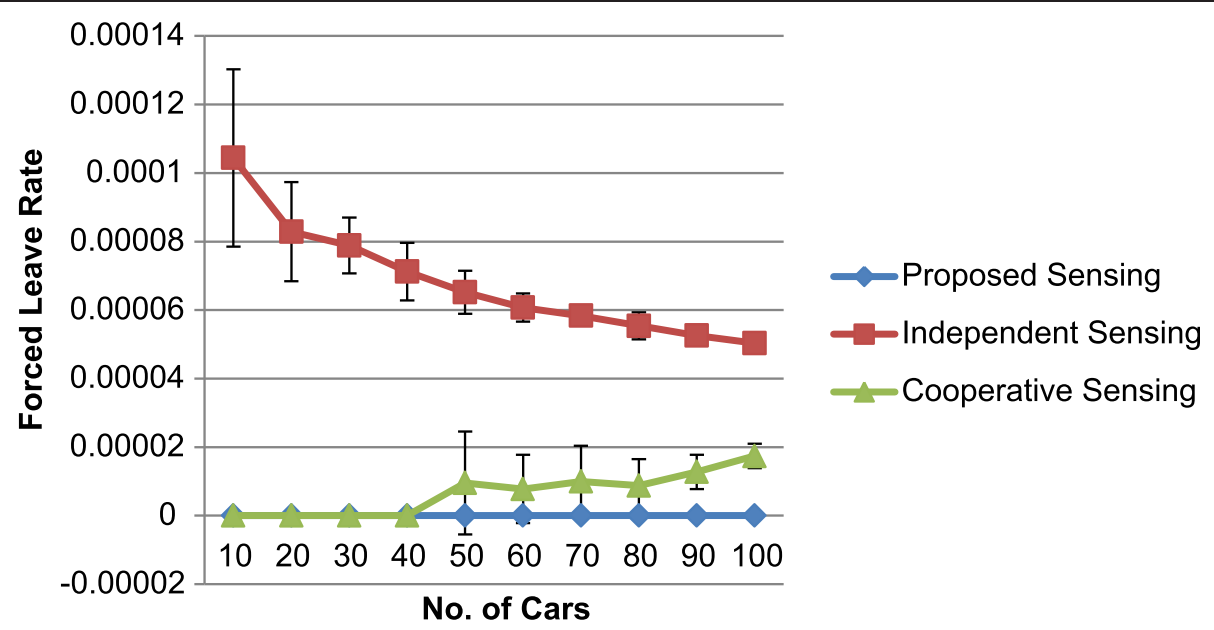

Fig. 17 Forced leave ratio vs no. of cars

times the vehicles, once they acquire the channels, are forced to leave the channels due to the return of the primary user. A lower forced leave ratio indicates the fewer disturbances to the communication of the secondary users.

These metrics have been measured in a number of different scenarios: varying numbers of cars, number of $\mathrm{CR}$ spectrum channels, and varying average speeds of the vehicles, keeping all other factors constant. Results observed for allocation rate are given by graphs in Figs. 8, 9, and 10. It is clear from the graphs that the allocation rate for our proposed algorithm is better as compared to the independent sensing and cooperative sensing mechanisms. Results obtained for false alarm ratio are given in Figs. 11, 12, and 13. Results show that the false alarm ratio of our proposed algorithm is better than the independent sensing mechanism, but cooperative sensing is better in this respect. As false alarms are not harmful, so it is not a problem if we obtain a higher allocation rate, a lower rejection rate, and a lower forced leave ratio. Figures 14, 15, and 16 show the rejection rate graphs. It is evident from the graphs that our proposed algorithm has a lower rejection rate compared to the other two mechanisms. Figures 17, 18, and 19 indicate the forced leave ratio. The forced leave ratio of our proposed mechanism is 0 . This is due to the fact that our proposed algorithm monitors the channels and calculates the higher probability of availability for the duration of the intended duration of the transmission. Hence, it provides the list of those channels which will be available for a longer duration calculated based on the history. All the graphs show the error bars specifying standard deviation. Error bars not visible on some graphs are too small to be displayed.

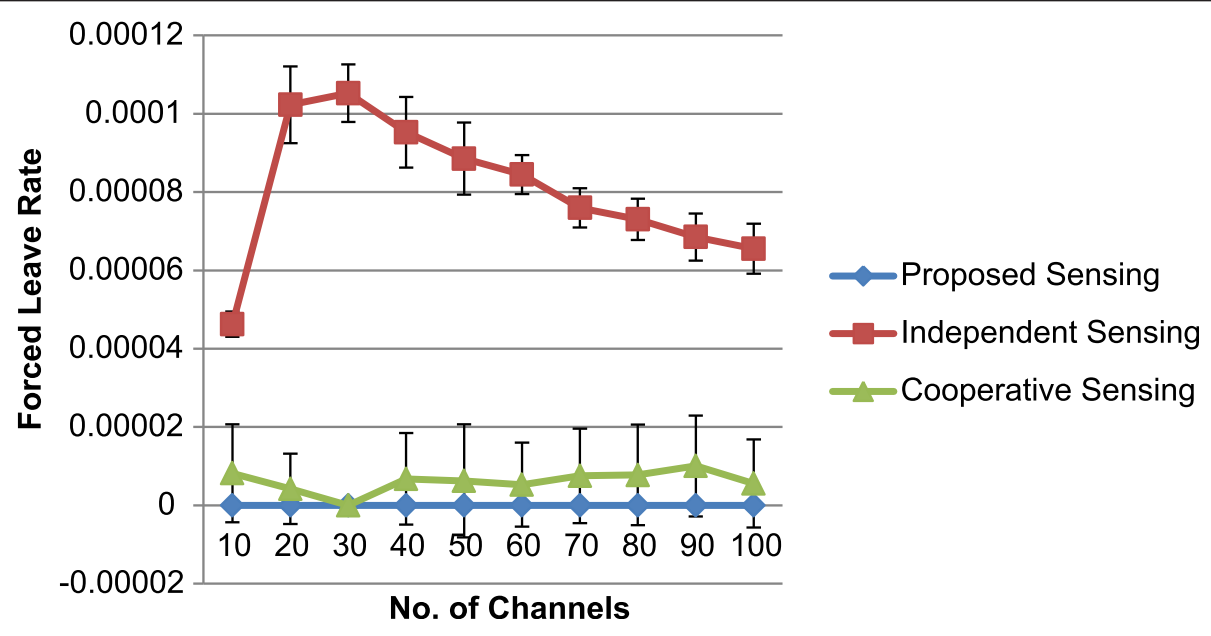

Fig. 18 Forced leave ratio vs no. of channels 


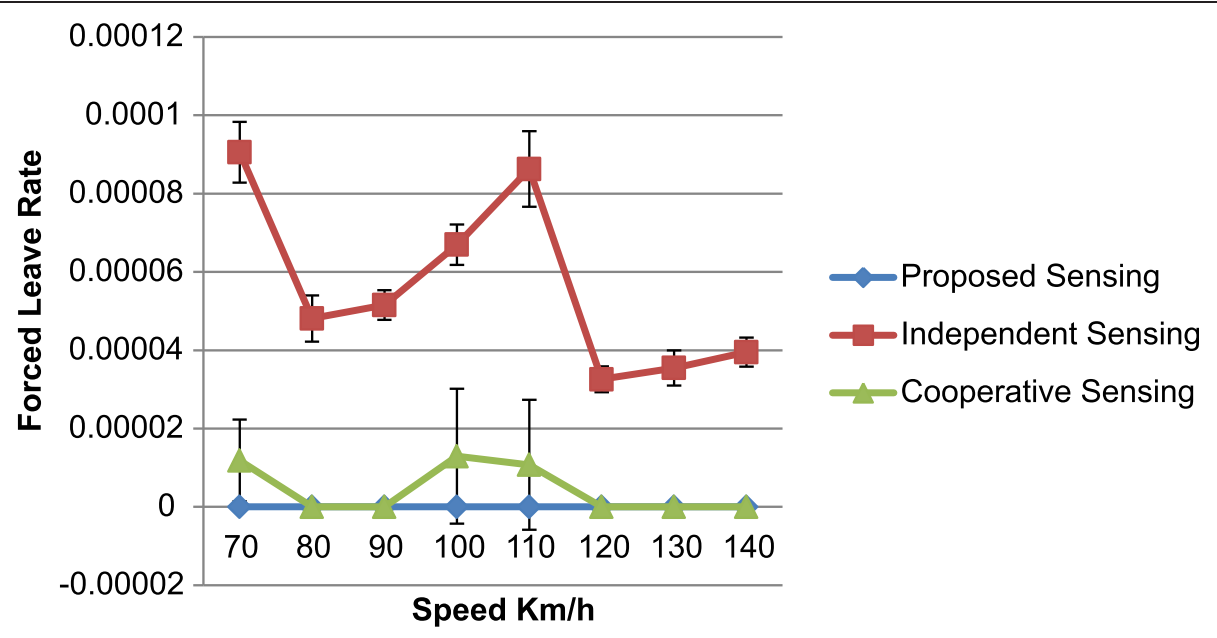

Fig. 19 Forced leave ratio vs speed

\section{Conclusions}

Day by day, the vehicular traffic is increasing on the roads; so it is the need of the hour to search for the vacant frequency slots in CR spectrum and then utilize until these are occupied again by the primary users. Our proposed algorithm is based on the historic data of sensing the CR spectrum, so it provides a clear picture of spatio-temporal and frequency slots for its future activity. It observes the primary user's activity and timing related to acquiring and leaving the channel for a particular distance slot and frequency. Based on these computations and observations, a list is prepared giving priority to the channels which are most likely to be available for the duration of the intended transmission. The simulation results obviously show the success of our algorithm. In the future, we intend to work on spectrum management and specifically more on allocation of the spectrum to secondary users based on the sensing results achieved so far in this paper. We also intend to work on utilization of spectrum by the secondary users and vacation of the spectrum in case the primary user returns.

\footnotetext{
Abbreviations

DRSU: directional road side unit; $N_{\mathrm{M}}$ : main coordinator; $N_{\mathrm{F}}$ : front coordinator; $N_{R}$ : back coordinator; $S_{C}$ : current distance segment; $S_{b c}$ : third last distance segment of backward cluster; $S_{\mathrm{fc}}$ : third distance segment of front cluster; $N_{\mathrm{j}}$ : current vehicle; CCH: control channel; DSRC: dedicated short-range communication; GPS: Global Positioning System; $T_{s}$ : sensing time interval; Re\{.\}: real part of the received complex waveform; $f_{*}^{i}$ : carrier frequency of the primary channel; $f_{s}^{i}$ : pilot carrier frequency of the secondary user; $S_{\mathrm{LP}}(t)$ : equivalent low-pass representation of the detected primary or secondary user signal; $N_{\mathrm{LP}}(t)$ : additive white Gaussian noise with 0 mean; $V_{\text {id: }}$ vehicle identity; $X Y$ : $x$ and $y$ coordinates; $C H$ : $C R$ channel; RES: result sensed; $T_{k}$ : time slot; $P_{a v}$ : probability of availability; $s$ : number of time intervals; $K$ : number of time slots; BSS: basic service set.
}

\section{Competing interests}

The authors declare that they have no competing interests.

\section{Author details}

${ }^{1}$ Electrical Engineering Department, Air University, E-9, Islamabad, Pakistan. ${ }^{2}$ LMKR, Ufone Tower, Blue Area, Islamabad, Pakistan.

Received: 18 June 2014 Accepted: 1 June 2015

Published online: 10 June 2015

\section{References}

1. World Health Organization, Global status report on road safety, 2013.

2. C. Y. Yeun,CY Yeun, M Al-Qutayri, F. Al-Hawi, Efficient Security Implementation for Emerging VANETs, UbiCC J. 4(4), (2009)

3. G Abdalla, MA Abu-Rgheff, SM Senouci, Current Trends in Vehicular Ad Hoc Networks (UbiCC Journal - Special Issue, March, 2008)

4. Wikipedia dedicated short-range communications, http://en.wikipedia.org/ wiki/Dedicated_short-range_communications.

5. M Zeeshan Hameed, F Fethi, LTE and IEEE 802.11p for vehicular networking: a performance evaluation. EURASIP J. Wirel. Commun. Netw. 2014, 89 (2014)

6. John B. Kenney, Dedicated Short-Range Communications (DSRC) Standards in the United States, Proc. IEEE: 99(7), (July 2011)

7. Department for transport, government of UK, road traffic statistics, (2013)

8. Federal communications commission, spectrum policy task force, Rep. ET Docket no. 02-135, (Nov. 2002).

9. J Mitola, GQ Maguire, Cognitive radio: making software radios more personal. IEEE Personal Commun. 6, 13-18 (1999)

10. S Haykin, Cognitive radio: brain-empowered wireless communications. IEEE J. Sel. Areas Commun. 23, 201-220 (2005)

11. W Zhang, RK Mallik, K Letaief, Cooperative Spectrum Sensing Optimization in Cognitive Radio Networks (IEEE International Conference on, Communications, 2008). Pp 3411-3415

12. K Fawaz, A Ghandour, Improving Reliability of Safety Applications in Vehicle Ad hoc Networks Through the Implementation of a Cognitive Network. In the Proceedings of the 17th International Conference on Telecommunications, (2010) p. 798-805

13. MD Felice, and KR Chowdhury, Analyzing the Potential of Cooperative Cognitive Technology on Inter-Vehicle Communication. In the Proceedings of the 2010 IFIP Wireless Days, (Venice, 2010) p. 1-6

14. Wooseong Kim, Soon Y Oh, CoRoute: A New Cognitive Anypath Vehicular Routing Protocol. In the Proceedings of the 2011 7th International Wireless Communications and Mobile Computing Conference, (2011) p.766-771

15. L Husheng, DK Irick, Collaborative Spectrum Sensing in Cognitive Radio Vehicular Ad hoc Networks: Belief Propagation on Highways. In the Proceedings of the 2010 71st IEEE Vehicular Technology Conference, (Taipei, 2010), p. 1-5

16. E R Nyanhete, Mzyece, K Djouani, Operation and performance of cognitive radios in vehicular environments. Tshwane University of Technology (2011) 
17. D B Rawat, Y Zhao, G Yan, CRAVE: Cognitive Radio Enabled Vehicular Communications in Heterogeneous Networks. In the Proceedings of the 2013 IEEE Radio and Wireless Symposium, (2013) p. 190-192

18. M D Felice, K R Chowdhury, L Bononi, Analyzing the potential of cooperative cognitive radio technology on inter-vehicle communications, IFIP Wireless Days (WD), (2010), p.1-6

19. MD Felice, KR Chowdhury, L Bononi, Cooperative spectrum management in cognitive vehicular ad hoc networks, IEEE Vehicular Networking Conference (VNC), 2011, pp. 47-54

20. Z Chenglin, S Mengwei, L Bin, Z Long, P Xiao, Blind spectrum sensing for cognitive radio over time-variant multipath flat-fading channels. EURASIP J. Wirel. Commun. Netw. 2014, 84 (2014)

21. $Y$ Xiao, $\mathrm{P}-\mathrm{HH}$ Wang, A novel sensing coordination framework for CR-VANETs. IEEE Trans. Vehicular Technol. 59(4), 1936-1948 (2010)

22. I H Brahmi, S Djahel, Y G Doudane, A Hidden Markov Model Based Scheme for Efficient and Fast Dissemination of Safety Messages in VANETs. In the Proceedings of the 2012 IEEE Global Communication Conference, (2012) p. 177-182

23. AR Biswas, TC Aysal, S Kandeepan, Cooperative Spectrum Sensing for Dynamic Cognitive Radio Networks, in In the Proceedings of the 2009 IEEE International Conference on Communications, Dresden, 2009, pp. 1-5

24. F Riaz, I Shafi, SF Hasan, W Younas, Y Mehmood, Vehicle-to-Vehicle Communication Enhanced by Cognitive Approach and Multi-Radio Technologies, in In the Proceedings of the 2012 IEEE International Conference on Emerging Technologies, Islamabad, 2012, pp. 1-6

25. G Ganesan, Y Li, Cooperative spectrum sensing in cognitive radio, part I: two user networks. IEEE Trans. Wirel. Commun. 6(6), 2204-2213 (2006)

26. SH Abbassi, IM Qureshi, O Khalid, H Abbasi, Basic structural change in vehicular adhoc networks. J. Netw. Technol. 3(4), 149-154 (2013)

27. SH Abbassi, IM Qureshi, H Abbasi, Performance of uni-directional road side units in vehicular adhoc networks, International Conference on Network Computing and Applications, 2014

28. S. H. Abbassi, I. M. Qureshi, B. R. Alyaei, H. Abbasi, K. Sultan, An efficient spectrum sensing mechanism for CR-VANETs, J. Basic Appl. Sci. Res: 3(12), 2013

\section{Submit your manuscript to a SpringerOpen ${ }^{\circ}$ journal and benefit from:}

- Convenient online submission

- Rigorous peer review

- Immediate publication on acceptance

- Open access: articles freely available online

- High visibility within the field

- Retaining the copyright to your article

Submit your next manuscript at $>$ springeropen.com 